\title{
DETERMINING ANALYTICITY FOR PARAMETER OPTIMIZATION OF THE GEGENBAUER RECONSTRUCTION METHOD*
}

\author{
A. GELB $^{\dagger}$ AND Z. JACKIEWICZ
}

\begin{abstract}
The Gegenbauer reconstruction method effectively eliminates the Gibbs phenomenon and restores exponential accuracy to the approximations of piecewise smooth functions. Recent investigations show that its success depends upon choosing parameters in such a way that the regularization and the truncation error estimates are equally considered. This paper shows that the underlying analyticity of the function in smooth regions plays a critical role in the regularization error estimate. Hence we develop a technique that first analyzes the behavior of the function in its regions of smoothness and then applies this knowledge to refine the regularization error estimate. Such refinement yields better parameter choices for the Gegenbauer reconstruction method, and is confirmed both by better accuracy and more robustness in the approximation of piecewise smooth functions.
\end{abstract}

Key words. Fourier pseudo-spectral approximation, Gegenbauer reconstruction, exponential convergence.

AMS subject classifications. 42A25, 42A30, 65D10

1. Introduction. The Gegenbauer reconstruction method, originally developed in [14], effectively eliminates the Gibbs phenomenon from the spectral approximation of a piecewise smooth function while maintaining its exponential convergence properties, even up to the discontinuities of the function. Since its conception, investigators have considered various aspects of the Gegenbauer reconstruction method, including construction from Fourier and other spectral expansion coefficients (e.g. Chebyshev and Legendre) in one and two dimensions as well as on spheres. Recent advancements of the Gegenbauer reconstruction method include an adaptation to noisy environments [2], avoidance of round-off error [7], parameter optimization techniques [7] and [15], and an alternative implementation by the inverse method [17]. All have contributed to making the Gegenbauer reconstruction method a viable option in both high and low resolution environments for multi-dimensional and multi-scale problems, even in the presence of noise. Additionally, the Gegenbauer reconstruction method has been successfully employed in numerous scientific applications, including medical image reconstruction (e.g. [1] [10]) and as a post-processing technique for conservation laws (e.g. [8], [16]). A good overview of the development and application of the Gegenbauer reconstruction method can be found in [11], [12] and [13], and the references therein.

The success of the Gegenbauer reconstruction method hinges on the underlying analytic behavior of the piecewise smooth function $f(x)$ in the regions between known discontinuities. In particular, the approximation of the regularization error of the Gegenbauer reconstruction method has been guided by an assumption of smoothness, first described in [14] as:

*This work was partially supported by NSF grants DMS 0324957, DMS-0107428, and EAR0222327, and NIH-EB02533-01 (AG) and NSF grant DMS-9971164 (ZJ).

${ }^{\dagger}$ Department of Mathematics, Arizona State University, Tempe, Arizona 85287, e-mail: ag@math.la.asu.edu

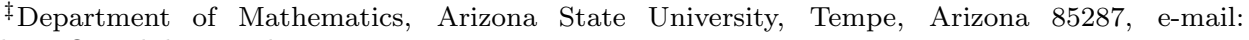
jackiewi@math.la.asu.edu 
Assumption 1.1. Let $f(x)$ be an analytic but non-periodic function on $[-1,1]$. There exist constants $\rho \geq 1$ and $C(\rho)$ such that for every $k \geq 0$,

$$
\max _{-1 \leq x \leq 1}\left|\frac{d^{k} f(x)}{d x^{k}}\right| \leq C(\rho) \frac{k !}{\rho^{k}} .
$$

The authors proceed to explain that this is a standard assumption for analytic functions where $\rho$ is the distance from $[-1,1]$ to the nearest singularity of $f(x)$ in the complex plane. Note that for $x \in[a, b]$ the assumption can be easily modified.

Later in [12] the regularization error is validated for functions under the following assumption:

ASSUMPTION 1.2. There exists a constant $0 \leq r_{0}<1$ and an analytic extension of $f(x)$ onto the elliptic domain

$$
D=\left\{z: \quad z=\frac{1}{2}\left(r e^{i \theta}+\frac{1}{r} e^{-i \theta}\right), \quad 0 \leq \theta \leq 2 \pi, \quad r_{0} \leq r<1\right\} .
$$

Once again this assumption is satisfied by all analytic functions defined on $[-1,1]$ and can easily be modified for $f(x)$ on general $[a, b]$.

Despite the fact that the smoothness of $f(x)$ is generally unknown, the Gegenbauer reconstruction method was shown to yield exponential convergence in most cases [14]. However, the accuracy of the method was seemingly function dependent, and varied with the particular parameters used in its construction.

A parameter optimization technique was first suggested for the Fourier based Gegenbauer reconstruction method in [7], where an 'average case scenario' $\rho=1$ in Assumption 1.1 was used to estimate the regularization error, since the underlying analyticity of the function in smooth regions was unknown. Later in [15] the procedure was refined for the Chebyshev case, where Assumption 1.2 was also considered. Here it was assumed that the smoothness of $f(x)$ was known explicitly, or equivalently that the values $\rho$ and $r_{0}$ were somehow pre-determined. As discussed in [15], such knowledge yields significant improvement to the sharpness of the regularization error estimate, which in turn produces a more refined technique for choosing optimal parameters for the reconstruction. It was shown that in some cases the reconstruction was dramatically improved, in particular when $\rho \neq 1$. It is therefore of critical interest to assess the analyticity of the underlying function for reconstruction, since such knowledge will serve to both refine and automate the parameter optimization techniques introduced in [7] and [15]. Specifically, we seek to accurately determine the smoothness parameters $\rho$ and $r_{0}$ in Assumptions 1.1 and 1.2. Once this is accomplished, the parameters for the Gegenbauer reconstruction method can be determined accordingly. The Gegenbauer reconstruction method can then be directly applied in any region of smoothness without further consideration of the behavior of the function.

Our paper is organized in the following way: In $\S 2$, the Gegenbauer reconstruction method based on Fourier coefficients is briefly reviewed. A general parameter optimization technique is described in $\S 3$, and a new method for evaluating the analyticity of $f(x)$ is introduced in $\S 4$. Numerical examples for the completely automated procedure are provided in $\S 5$. For ease of presentation, we restrict our discussion throughout this paper to the reconstruction of a smooth non-periodic function $f(x)$ on $[-1,1]$ from its Fourier coefficients, and note that although some additional complications involving edge detection will arise for piecewise smooth functions, the techniques discussed here for the specific purpose of reconstruction remain the same. 
2. Review of the Gegenbauer reconstruction method. Consider an analytic but non-periodic function $f(x)$ defined on the interval $[-1,1]$. The Fourier partial sum,

$$
f_{N}(x)=\sum_{n=-N}^{N} \widehat{f}_{n} e^{i n \pi x},
$$

with Fourier coefficients $\widehat{f}_{n}$ defined by

$$
\widehat{f}_{n}=\frac{1}{2} \int_{-1}^{1} f(x) e^{-i n \pi x} d x
$$

shows $O(1)$ spurious oscillations near the boundaries \pm 1 . Additionally, the convergence of $f_{N}(x)$ to $f(x)$ on $[-1,1]$ is reduced to first order. This behavior is the well known Gibbs phenomenon, and many techniques have been developed to reduce or eliminate its effects. The Gegenbauer reconstruction method, first introduced in [14], uses information from the first $2 N+1$ Fourier coefficients $(2.2)$ to completely resolve the Gibbs phenomenon and yields exponential convergence to $f(x)$ in the uniform norm on $[-1,1]$. Here we briefly describe the method and refer the reader to [14], [11], [12] and [13] for more complete details.

Denote by $C_{l}^{\lambda}$ the Gegenbauer polynomials [3], [5], i.e., the polynomials which are orthonormal with respect to the inner product,

$$
\left\langle C_{k}^{\lambda}, C_{l}^{\lambda}\right\rangle=\frac{1}{h_{l}^{\lambda}} \int_{-1}^{1}\left(1-x^{2}\right)^{\lambda-\frac{1}{2}} C_{k}^{\lambda}(x) C_{l}^{\lambda}(x) d x
$$

where for $\lambda>0$ the scaling factor $h_{l}^{\lambda}$ is defined by

$$
h_{l}^{\lambda}=\sqrt{\pi} C_{l}^{\lambda}(1) \frac{\Gamma\left(\lambda+\frac{1}{2}\right)}{\Gamma(\lambda)(l+\lambda)}, \quad C_{l}^{\lambda}(1)=\frac{\Gamma(l+2 \lambda)}{l ! \Gamma(2 \lambda)} .
$$

The Gegenbauer reconstruction method consists of approximating the function $f(x)$ by computing the truncated approximate Gegenbauer series

$$
f_{g}^{m, \lambda}(x)=\sum_{l=0}^{m} \widehat{g}^{\lambda}(l) C_{l}^{\lambda}(x),
$$

where the coefficients $\widehat{g}^{\lambda}(l)$ are given by

$$
\widehat{g}^{\lambda}(l)=\frac{1}{h_{l}^{\lambda}} \int_{-1}^{1}\left(1-x^{2}\right)^{\lambda-\frac{1}{2}} f_{N}(x) C_{l}^{\lambda}(x) d x .
$$

As first shown in [14], the coefficients (2.4) provide an exponentially accurate approximation to the exact Gegenbauer coefficients, defined by

$$
\widehat{f}^{\lambda}(l)=\frac{1}{h_{l}^{\lambda}} \int_{-1}^{1}\left(1-x^{2}\right)^{\lambda-\frac{1}{2}} f(x) C_{l}^{\lambda}(x) d x,
$$

provided that $\lambda, m \sim N$, that is, they scale like $N$. In fact, if $\lambda$ and $m$ are proportional to $N$, i.e.,

$$
\lambda=\alpha N, \quad m=\beta N,
$$


then

$$
\max _{-1 \leq x \leq 1}\left|f(x)-f_{g}^{m, \lambda}(x)\right| \leq A\left(q_{T}^{N}+q_{R}^{N}\right),
$$

where $A$ is a polynomial in $N, q_{T}$ corresponds to the truncation error

$$
T E=\max _{-1 \leq x \leq 1}\left|\sum_{l=0}^{m}\left(\widehat{f}^{\lambda}(l)-\widehat{g}^{\lambda}(l)\right) C_{l}^{\lambda}(x)\right|,
$$

and $q_{R}$ corresponds to the regularization error

$$
R E=\max _{-1 \leq x \leq 1}\left|f(x)-\sum_{l=0}^{m} \hat{f}^{\lambda}(l) C_{l}^{\lambda}(x)\right| .
$$

Both $q_{T}$ and $q_{R}$ are constants less than one. Although it was shown that in general $\lambda, m \sim N$ will yield exponential convergence [14], the parameter choices (2.6) lead to the convenient analysis of (2.8) and (2.9) and are furthermore shown in [7] to produce greater theoretical accuracy than other parameter alternatives.

It was shown in [14] that the constant $q_{T}$ for (2.6) takes the form

$$
q_{T}=\frac{(\beta+2 \alpha)^{\beta+2 \alpha}}{(2 \pi e)^{\alpha} \alpha^{\alpha} \beta^{\beta}} .
$$

As discussed in the introduction, the constant $q_{R}$, representing the regularization error, can be defined in different ways depending on the smoothness of $f(x)$ on $[-1,1]$. If we consider the latter case given in Assumption 1.2, then $q_{R}$ is defined as [12]:

$$
q_{R}=q_{R}^{1}=\left(\frac{(1+2 \gamma)^{\frac{1+2 \gamma}{2}}}{(2 \gamma)^{\gamma}} r_{0}\right)^{\beta}, \quad \gamma=\frac{\lambda}{m},
$$

The parameters defined in (2.6) yield

$$
q_{R}^{1}=\frac{(\beta+2 \alpha)^{\frac{\beta+2 \alpha}{2}} r_{0}^{\beta}}{(2 \alpha)^{\alpha} \beta^{\frac{\beta}{2}}} .
$$

On the other hand, Assumption 1.1, gives the ratio $q_{R}$ as [14]:

$$
q_{R}=q_{R}^{2}=\left(\frac{(1+2 \gamma)^{1+2 \gamma}}{\rho 2^{1+2 \gamma} \gamma^{\gamma}(1+\gamma)^{1+\gamma}}\right)^{\beta}, \quad \gamma=\frac{\lambda}{m},
$$

which assuming (2.6) reduces to

$$
q_{R}^{2}=\frac{(\beta+2 \alpha)^{\beta+2 \alpha}}{2^{\beta+2 \alpha} \alpha^{\alpha}(\alpha+\beta)^{\alpha+\beta} \rho^{\beta}} .
$$

In $\S 3$ we propose a parameter optimization technique based on the minimization of the ratio (2.10) corresponding to the truncation error assuming that it is equal to the respective ratios (2.12) and (2.14) corresponding to the regularization errors. We note that although the original theoretical discussion [14] is based on the continuous 
Fourier coefficients (2.2), all of our numerical experiments are conducted utilizing the corresponding pseudo-spectral Fourier coefficients

$$
\widetilde{f}_{n}=\frac{1}{2 N c_{n}} \sum_{j=0}^{2 N} f\left(x_{j}\right) e^{-i n \pi x_{j}},
$$

based on equally spaced grid points $x_{j}, j=0,1, \ldots, 2 N$. Here $c_{n}=\frac{1}{2}$ for $n=-N$ or $n=N$, and $c_{n}=1$ otherwise.

3. Determining optimal parameters. Although it was shown in [14] that the parameter choices $\lambda=\alpha N$ and $m=\beta N$ appearing in (2.6) yield exponential convergence of $f_{g}^{m, \lambda}(x)$ to $f(x)$ for a large class of functions, the accuracy of the approximation, and even the convergence itself, can depend critically on the choices of these parameters. For instance, numerical experiments in [14] for the reconstruction of analytic non-periodic functions from Fourier coefficients indicated that choosing the parameters $\alpha=\beta=0.4$ typically led to more accurate results than for $\alpha=\beta=0.25$, provided that no round-off error was present. It was additionally shown that for fixed $\beta \leq \frac{\pi}{4}$, the ratio $q_{T}$ defined by (2.10) attains its minimum for

$$
\alpha=\frac{1}{4}(\pi-2 \beta+\sqrt{\pi(\pi-4 \beta)}) .
$$

The resulting experiments conducted with $\beta=0.25$ and $\alpha$ chosen according to (3.1) demonstrated very fast convergence for functions having no regularization error. However, for more general functions in which both the truncation and regularization errors are present, the convergence rate was not improved. This was further analyzed in [7] where a systematic approach was designed to select $m$ and $\lambda$ to yield exponential convergence for both the truncation and regularization errors. A similar technique was later developed for the Chebyshev-Gegenbauer reconstruction method in [15], where it was additionally assumed that the underlying "smoothness" behavior of $f(x)$ in regions between the discontinuities was known, i.e. the values of $\rho$ and $r_{0}$ in Assumptions 1.1 and 1.2 were given. It was also shown that if the parameters $m$ and $\lambda$ were characterized by (2.6), then the contribution of $A$ to the error bound on the right hand side of (2.7) was negligible since $A$ is only a polynomial in $N$. Hence the optimization technique presented in [15] is a function only of the parameters $\alpha$ and $\beta$, and is independent of $N$. We will follow this approach here.

The strategy for choosing $\alpha$ and $\beta$ in (2.6) is simple. As in [7] and [15], it is based on balancing the truncation (2.10) and regularization errors (2.12) or (2.14). Specifically, we set $q_{T}=q_{R}^{1}$ or $q_{T}=q_{R}^{2}$, depending on the underlying smoothness of the function. The optimal parameters are then determined by the minimization of $q_{T}$.

We realize that this optimization is valid only for relatively small $N$ or sparse data sets. Round-off error greatly impacts the ability of the computed Gegenbauer coefficients to decay at the rate needed to balance the large growth of the Gegenbauer polynomials. Here we proceed with the discussion without consideration of roundoff error, and note that some numerical results may be more impacted by round-off error than by lack of analytical convergence. In such cases it was shown in [7] that rather than forcing the relationship (2.6) of $m$ and $\lambda$ with $N$, it is better to determine maximum admissible values for $m$ and $\lambda$ such that the Gegenbauer reconstruction is only minimally affected by round-off error. All admissible combinations $m$ and $\lambda$ are then considered in the computation of (2.7), and the smallest value of (2.7) yields 
the "optimal" admissible pair of $m$ and $\lambda$. Details are discussed in [7]. However, the underlying smoothness of a function still plays a critical role in determining suitable parameters even when round-off error becomes relevant in the reconstruction.

To illustrate the parameter optimization technique derived here, Figure 3.1 displays the contours of $q_{T}$ (dash-dotted lines), $q_{R}^{1}$ (dotted lines), and the curve $q_{T}=q_{R}^{1}$ for the given value $r_{0}=\sqrt{2}-1$ (solid line). For comparison with the previous parameter selection strategies, Figure 3.1 also displays the curve defined by (3.1) (dashed line).

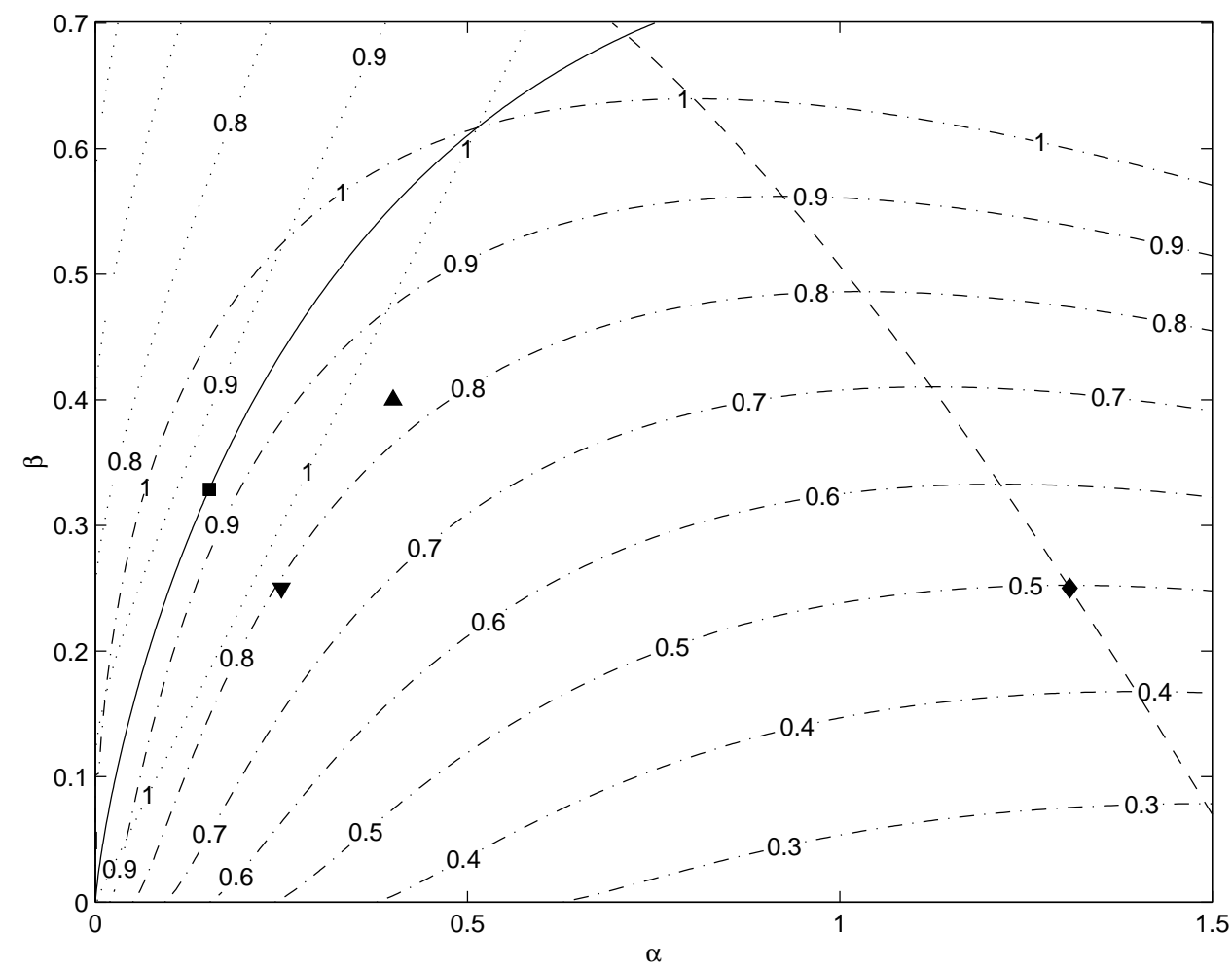

FIG. 3.1. Contour plots of $q_{T}$ (dash-dotted lines), $q_{R}^{1}$ (dotted lines), the curve $q_{T}=q_{R}^{1}$ for $r_{0}=\sqrt{2}-1$ (solid line), and the curve defined by (3.1) (dashed line). Specific point values for $\alpha$ and $\beta$ include $\alpha=\beta=0.25$ (downward triangle), $\alpha=\beta=0.4$ (upward triangle), $\beta=0.25$ and $\alpha=1.31$ computed by (3.1) (diamond), and the points obtained using our strategy based on minimization of (3.2) (square).

Figure 3.2 displays the corresponding contour plots of $q_{R}^{2}$ for the 'average case scenario', i.e., with $\rho=1$ which, as discussed in [12] and [15], corresponds to $r_{0}=\sqrt{2}-1$ assuming that the singularity of $f(x)$ closest to the interval $[-1,1]$ is located on the imaginary axis. Both figures display the values for $\alpha$ and $\beta$ originally suggested in [14] to illustrate the relationship of the parameters to the truncation and regularization errors. Here we point out that the strategy proposed in this paper takes into account both the regularization and truncation errors obtained from the Gegenbauer reconstruction method. These values are obtained numerically by minimizing the objective functions

$$
\phi^{l}(\alpha, \beta)=q_{T}+C\left(q_{T}-q_{R}^{l}\right)^{2}, \quad l=1,2,
$$


where $C$ is a positive penalty constant which was chosen as $C=1000$. The resulting values for $q_{T}=q_{R}^{1}$ with $r_{0}=\sqrt{2}-1$ are $\alpha=0.176, \beta=0.343$, while the same algorithm for $q_{T}=q_{R}^{2}$ with $\rho=1$ yields $\alpha=0.370, \beta=0.401$.

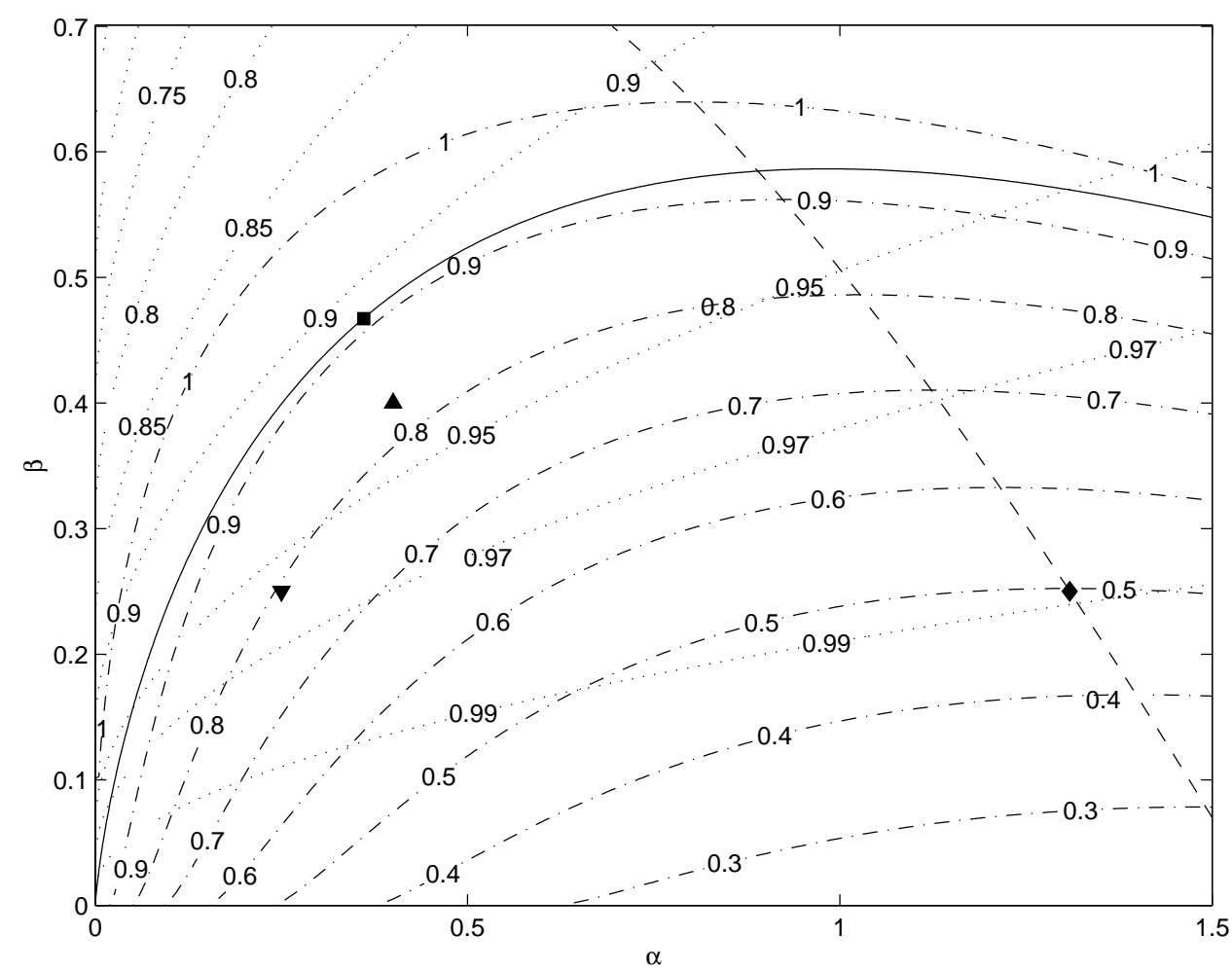

FIG. 3.2. Contour plots of $q_{T}$ (dash-dotted lines), $q_{R}^{2}$ (dotted lines), the curve $q_{T}=q_{R}^{2}$ for $\rho=1$ (solid line), and the curve defined by (3.1) (dashed line). Specific point values for $\alpha$ and $\beta$ include $\alpha=\beta=0.25$ (downward triangle), $\alpha=\beta=0.4$ (upward triangle), $\beta=0.25$ and $\alpha=1.31$ computed by (3.1) (diamond), and the points obtained using our strategy based on minimization of (3.2) (square).

Figures 3.3 and 3.4 depict the optimal choices for $\alpha$ and $\beta$ obtained by minimizing (3.2) for $0 \leq r_{0} \leq 1$ and $\rho \geq 1$ (upper graphs) together with the corresponding values of $q_{T}=q_{R}^{l}, l=1,2$ (lower graphs). The thin lines plotted in Figure 3.4 illustrate the values of $\alpha$ and $\beta$ obtained by using (2.14) for $\rho<1$. Once $\rho$ is less than about 0.5 , the resulting estimates are meaningless since $q_{T}=q_{R}^{2}$ is complex.

There are several things to note here:

1. The optimization strategy (3.2) used here assumes knowledge of the smoothness parameters $\rho$ and $r_{0}$. This information is not inherently known, and hence in $\S 4$ we develop a technique to approximate these values to allow a more refined strategy for determining optimal parameters $\alpha$ and $\beta$.

2. We reiterate that the optimal strategy (3.2) does not consider round-off errors in its minimization. As discussed previously in [7], the Gegenbauer reconstruction method is numerically inhibited by large values of $m=\beta N$ and $\lambda=\alpha N$, due to the lack of numerical convergence of the Gegenbauer coefficients (2.4). For instance, the values for $\alpha$ shown in Figure 3.4 would be 

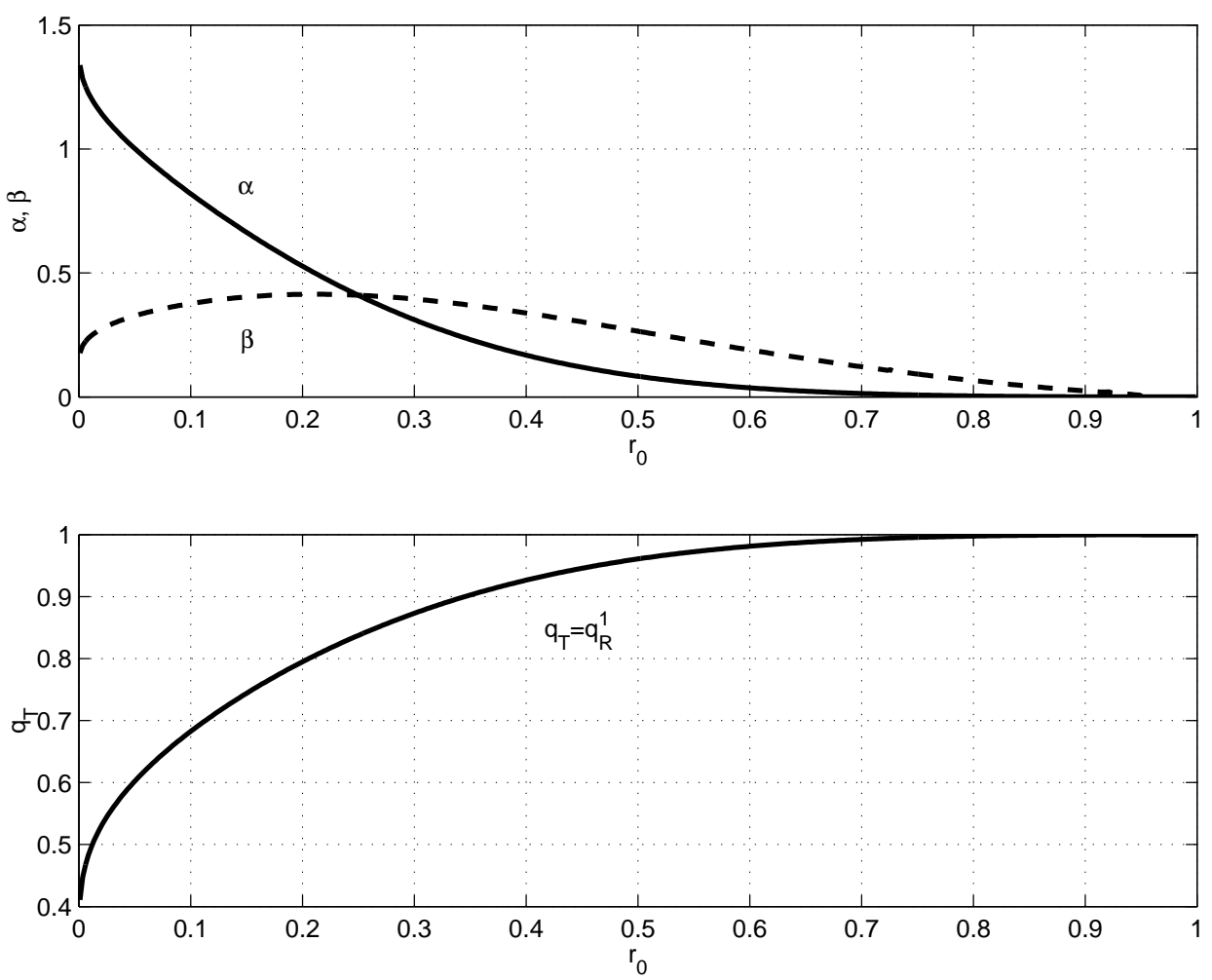

FIG. 3.3. Parameters $\alpha$ and $\beta$ versus $r_{0}$ (upper graph) and $q_{T}=q_{R}^{1}$ versus $r_{0}$ (lower graph).

unreasonable for $N>32$ (conservatively), and in general, the numerically computed Gegenbauer coefficients cease to decay at the required exponential rate for even moderate values of $\alpha$ and $\beta$.

3. Although the algorithm is derived for smooth functions in $[-1,1]$, it can be easily modified to optimize parameter choices for any smooth region $[a, b]$.

To compare the strategy based on minimizing $\phi^{1}(\alpha, \beta)$ or $\phi^{2}(\alpha, \beta)$, Figure 3.5 exhibits the equations $q_{T}=q_{R}^{1}$ and $q_{T}=q_{R}^{2}$ versus $r_{0}$ under the assumption that the closest singularity of $f(x)$ to $[-1,1]$ is on the imaginary axis. As explained in [15] (see also [12]), the relationship between $r_{0}$ and $\rho$ is then given by

$$
\rho=\frac{1}{2}\left(\frac{1}{r_{0}}-r_{0}\right) \quad \text { or } \quad r_{0}=\sqrt{1+\rho^{2}}-\rho .
$$

Figure 3.5 indicates that if both strategies for determining $\alpha$ and $\beta$ are applicable then minimizing $\phi^{2}(\alpha, \beta)$ will yield smaller analytical truncation and regularization errors than minimizing $\phi^{1}(\alpha, \beta)$, concurring with previous results described for the Chebyshev case in [15]. Figures 3.1 and 3.2 suggest the same conclusion. However, as apparent from the top of Figure 3.4 and prior remarks, minimizing $\phi^{2}(\alpha, \beta)$ for $\rho \geq 2$ $\left(0 \leq r_{0} \leq \sqrt{5}-2\right)$ can potentially yield large round-off errors in the Gegenbauer reconstruction case. In fact, the strategy can be inhibited by round-off error even when $\rho<2\left(r_{0}>\sqrt{5}-2\right)$ for $\lambda=\alpha N$ as $N$ increases. In this case, the top of Figure 3.3 suggests that the strategy based on minimizing $\phi^{1}(\alpha, \beta)$, although not theoretically optimal, will yield better results as $N$ increases. Additionally, note the 

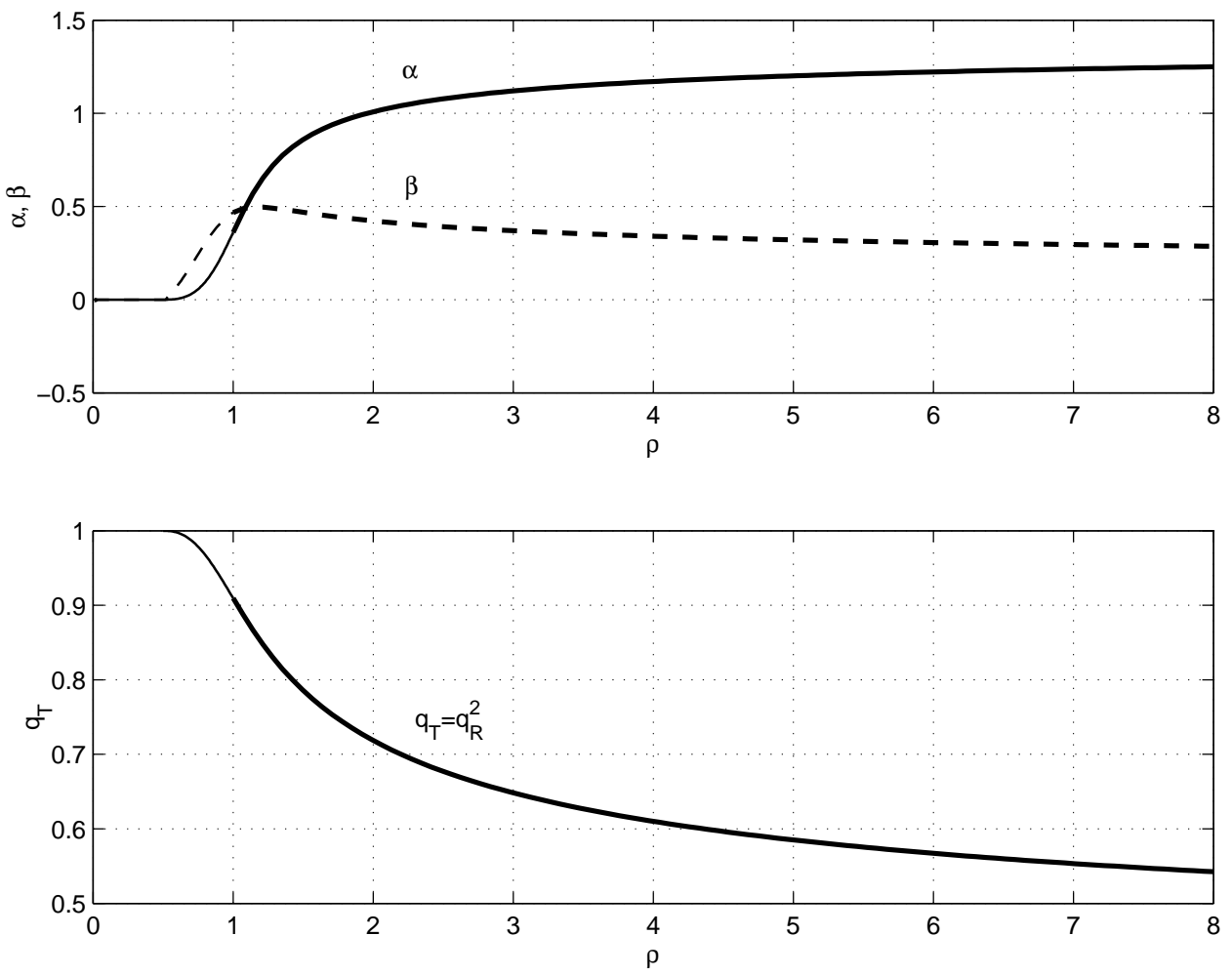

FIG. 3.4. Parameters $\alpha$ and $\beta$ versus $\rho$ (upper graph) and $q_{T}=q_{R}^{2}$ versus $\rho$ (lower graph).

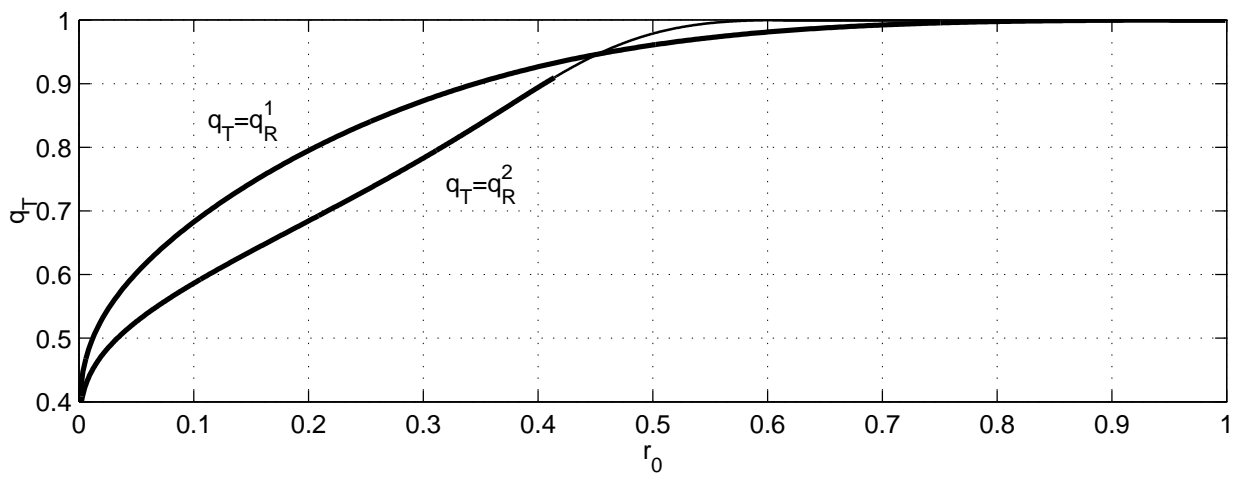

FIG. 3.5. Ratios $q_{T}=q_{R}^{1}$ and $q_{T}=q_{R}^{2}$ versus $r_{0}$.

thin line in Figure 3.5 corresponding to $\rho<1$ or equivalently $r_{0}>\sqrt{2}-1$. In this case, $\rho<0.5\left(r_{0}>0.618\right)$ produces complex values for $q_{T}=q_{R}^{2}$ and subsequently yields useless estimates.

Due to the the various factors discussed above, we will employ the parameters $\alpha$ and $\beta$ determined by minimizing $\phi^{1}(\alpha, \beta)$ in our numerical experiments discussed in $\S 5$. 
4. Determination of smoothness parameters. As discussed in $\S 3$, the notion of smoothness plays an important role in determining the optimal parameters $\alpha$ and $\beta$ for the Gegenbauer reconstruction method. Specifically we wish to determine the analyticity of the underlying function based on the parameters $\rho$ and $r_{0}$ described in Assumptions 1.1 and 1.2. These values are unknown in advance and must be approximated from the $2 N+1$ Fourier coefficients (2.2) or pseudo-spectral coefficients (2.15). This is an inherently difficult problem, since the Fourier approximation (2.1) is contaminated by Gibbs oscillations and cannot be expected to produce reliable results for determining factors $\rho$ or $r_{0}$.

As shown in [9], another way for assessing smoothness of a function is from its Chebyshev coefficients, namely

$$
\lim \sup _{k \rightarrow \infty}\left|a_{k}\right|^{1 / k}=r_{0}
$$

where $a_{k}, k=0,1, \ldots$, are the Chebyshev coefficients of the function $f(x)$. This suggests that determining the smoothness parameters $\rho$ and $r_{0}$ is equivalent to evaluating the decay rate of the Chebyshev coefficients $a_{k}$ as $k \rightarrow \infty$. Clearly (4.1) cannot be used directly since the exact function $f(x)$ is not known. However it is possible to compute an approximation to the Chebyshev coefficients $a_{k}^{(N)}$ based on the partial sum $f_{N}(x)$ in (2.1). Unfortunately, due to the poor convergence properties of $f_{N}(\mathrm{x})$, we cannot expect that

$$
r_{0}^{(N)}:=\lim \sup _{k \rightarrow \infty}\left|a_{k}^{(N)}\right|^{1 / k}
$$

will give a reliable estimate of $r_{0}$. Hence we seek a function approximation $\widetilde{f}_{N}(x)$ whose Chebyshev coefficients characterize the original function $f(x)$, such that

$$
\widetilde{r}_{0}^{(N)}:=\limsup _{k \rightarrow \infty}\left|\widetilde{a}_{k}^{(N)}\right|^{1 / k} \rightarrow r_{0},
$$

where $\widetilde{a}_{k}^{(N)}, k=0,1, \ldots, K$, are the computed Chebyshev coefficients of $\widetilde{f}_{N}(x)$.

One possibility is to compute $\tilde{f}_{N}(x)=f_{g}^{m, \lambda}(x)$ in (2.3) based on the assumed value of $\rho=1$, as in [7]. This approximation converges exponentially to $f(x)$ when $\rho \neq 1$ (although not optimally), yet the computed Chebyshev coefficients $\widetilde{a}_{k}^{(N)}$ will not yield the correct $r_{0}$ in (4.3). This is due to the fact that $f_{g}^{m, \lambda}$ is a polynomial yielding $r_{0}=\infty$ irrespective of $m$ and $\lambda$.

A second alternative is to exploit the Bernoulli reconstruction method, [4] and [6], to approximate a function that is characterized by the same analyticity of $f(x)$ once the discontinuities have been removed. To be more specific, in this approach we define

$$
\tilde{f}_{N}(x):=f_{N}(x)-\frac{\sigma_{N}}{2}\left(s_{N}(x)-s(x)\right),
$$

where $s_{N}(x)$ is the Fourier partial sum of the function

$$
s(x)=x, \quad-1 \leq x \leq 1
$$

and $\sigma_{N}$ is an approximation to the exact jump $\sigma=f(1)-f(-1)$ of the function $f(x)$. This approximation can be computed from the formula [18]

$$
\sigma_{N}=\frac{\pi}{2 \mu_{1}}\left(f_{N}\left(\xi_{N}\right)-f_{N}\left(\bar{\xi}_{N}\right)\right)
$$


where

$$
\mu_{1}=\int_{0}^{\pi} \frac{\sin t}{t} d t \approx 1.852, \quad \xi_{N}=\frac{2 N-1}{2 N}, \quad \text { and } \quad \bar{\xi}_{N}=\frac{1-2 N}{2 N} .
$$

It can be verified that

$$
\widetilde{f}_{N}(x)-f(x)=g_{N}(x)-g(x)+\frac{\sigma-\sigma_{N}}{2}\left(s_{N}(x)-s(x)\right),
$$

where

$$
g(x)=f(x)-\frac{\sigma}{2} s(x), \quad \text { and } \quad g_{N}(x)=f_{N}(x)-\frac{\sigma}{2} s_{N}(x) .
$$

The function $g(x)$ is periodic and continuous on $[-1,1]$ and as a result $g_{N}(x)$ is linearly convergent to $g(x)$ in the uniform norm. Since $\sigma_{N}$ is also linearly convergent to $\sigma$, [18], it follows from (4.6) that $\widetilde{f}_{N}(x)$ is linearly convergent to $f(x)$. Therefore, (4.4) can be viewed as a preliminary resolution of the Gibbs phenomenon. More precisely, since $\widetilde{f}_{N}(x)$ was obtained by subtracting the oscillatory behavior of $f_{N}(x)$ we can expect that $\widetilde{f}_{N}(x)$ and $f(x)$ share similar smoothness characteristics on the interval $[-1,1]$ described by the parameters $\rho$ or $r_{0}$. Hence we expect that the Chebyshev coefficients $\widetilde{a}_{k}^{(N)}, k=0,1, \ldots, K$, for $K \approx N$, of the function $\widetilde{f}_{N}(x)$ will provide an accurate and reliable estimate of $r_{0}$ in (4.1). To illustrate that this is indeed the case, Figure 4.1 exhibits the approximations of $r_{0}, r_{0}^{(N)}$ and $\widetilde{r}_{0}^{(N)}$ versus $\rho$ for the family of functions

$$
f(x)=\frac{e^{x}}{x^{2}+\rho^{2}}, \quad-1 \leq x \leq 1 .
$$

In this case, $f(x)$ has singularities on the imaginary axis at $x= \pm i \rho$ and the relationship between $r_{0}$ and $\rho$ is given by (3.3). The approximations to $r_{0}$ are computed from the respective formulae

$$
\begin{gathered}
r_{0} \approx \min \left\{\left|a_{k}\right|^{1 / k}: k_{0} \leq k \leq K\right\}, \\
r_{0}^{(N)} \approx \min \left\{\left|a_{k}^{(N)}\right|^{1 / k}: k_{0} \leq k \leq K\right\},
\end{gathered}
$$

and

$$
\widetilde{r}_{0}^{(N)} \approx \min \left\{\left|\widetilde{a}_{k}^{(N)}\right|^{1 / k}: k_{0} \leq k \leq K\right\}
$$

which are less susceptible to round-off errors than the approximations based on (4.1), (4.2) and (4.3). Here, $k_{0}$ is a small positive integer chosen to eliminate the influence of the first few Chebyshev coefficients on the estimate of $r_{0}$ and $K<N$. The exact value of $r_{0}$ given in (3.3) for $f(x)$ given in (4.7) is also plotted in Figure 4.1 by a thick solid line. It is evident that the approximation for $\widetilde{r}_{0}^{N}(4.10)$ converges to the desired value $r_{0}$. Figure 4.2 displays the approximations $r_{0}, r_{0}^{(N)}$, and $\widetilde{r}_{0}^{(N)}$ versus $\rho$ for the family of functions

$$
f(x)=\frac{e^{x}}{x^{2}-(1+\rho)^{2}}, \quad-1 \leq x \leq 1,
$$




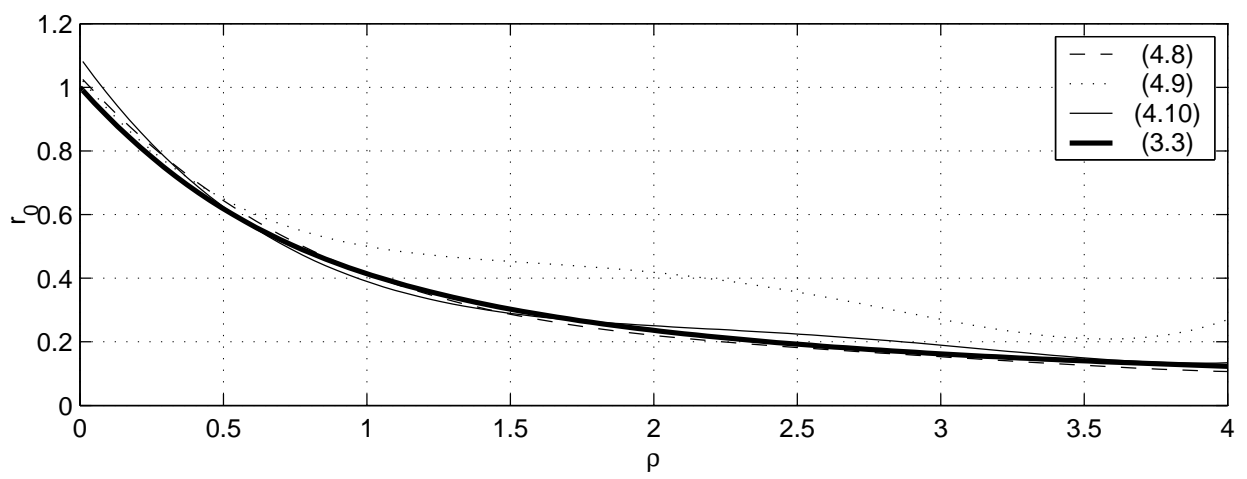

FIG. 4.1. $r_{0}, r_{0}^{(N)}$ and $\widetilde{r}_{0}^{(N)}$ given by (4.8), (4.9) and (4.10) for $K=N=20$ and $r_{0}$ given by (3.3) versus $\rho$ for the function (4.7).

which have singularities on the real axis at $x= \pm(1+\rho)$. In this case the relationship of $r_{0}$ and $\rho$ is given by ([12] and [15]):

$$
\rho=\frac{\left(r_{0}-1\right)^{2}}{2 r_{0}} \text { or } \quad r_{0}=1+\rho-\sqrt{\rho^{2}+2 \rho} .
$$

As exhibited in Figure 4.2, the value of $\widetilde{r}_{0}^{(N)}$ computed by (4.10) yields an accurate

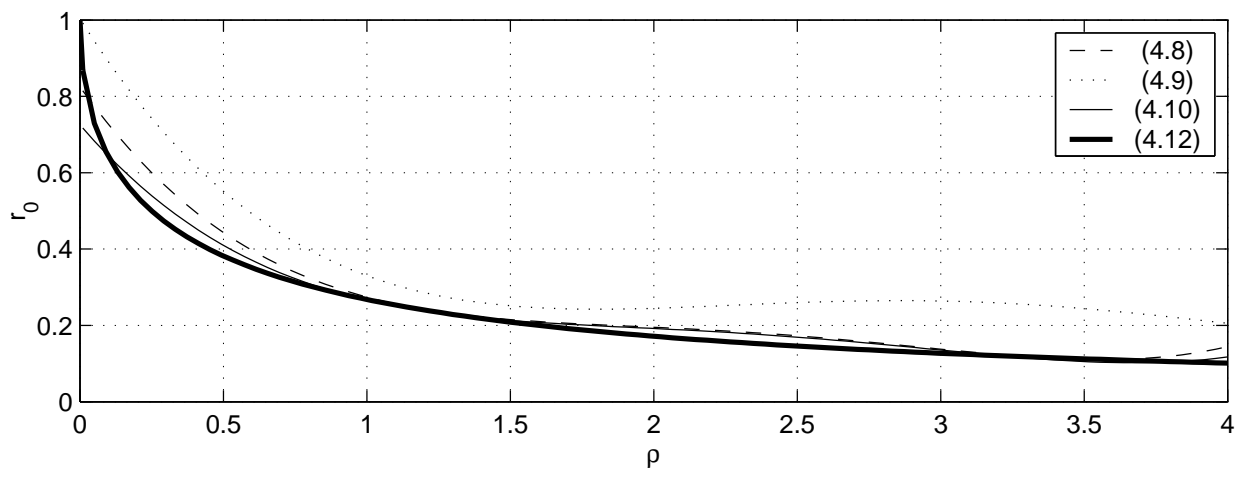

FIG. 4.2. $r_{0}, r_{0}^{(N)}$ and $\widetilde{r}_{0}^{(N)}$ given by (4.8), (4.9) and (4.10) for $K=N=20$ and $r_{0}$ given by (4.12) versus $\rho$ for the function (4.11).

comparison to (4.12), plotted by the thick solid line. The success of the smoothness predictor (4.10) will be further illustrated in $\S 5$.

It is important to consider the following:

1. Recall that when the closest singularity of $f(x)$ to $[-1,1]$ is on the imaginary axis, then the relationship between $r_{0}$ and $\rho$ is given by (3.3). This is further indication that parameter optimization based on Assumption 1.2, or equivalently the minimization of $\phi^{1}(\alpha, \beta)$ in (3.2), is more convenient since the computation of $r_{0}$ is possible without a-priori knowledge of the position of the closest singularities. To determine $\rho$ for $\phi^{2}(\alpha, \beta)$, it would first be necessary to determine where these singularities are in the complex plane. 
2. Observe in Figures 4.1 and 4.2 that $r_{0}$ is approximated for $K=20$. Although theoretically the estimate of $r_{0}$ should improve as $K \rightarrow \infty$, numerical implementation indicates that round-off error will affect the results for large $K$. Furthermore, reasonable estimates are already obtained for $K=20$ yielding better computational efficiency for the evaluation of the approximation to the parameter $r_{0}$.

3. As explained in [6], the approximation (4.4) to $g(x)$ can be of higher order. It is likely that the smoothness parameter $\widetilde{r}_{0}^{(N)}$ resulting from a higher order approximation of $g(x)$ will better match $r_{0}$ determined from (4.10). However, numerical experiments suggest that the first order approximation is good enough in resolving (4.3), and we will therefore confine ourselves to this approximation.

4. The method described is developed exclusively for one dimensional problems. Future investigations will address smoothness assessment for higher dimensional functions.

5. Numerical examples. In this section we demonstrate the effectiveness of using the optimal parameters $\alpha$ and $\beta$ which is determined by minimizing $\phi^{1}(\alpha, \beta)$ in (3.2). Recall that this optimization is inherently dependent on the smoothness parameter $r_{0}$ (4.8), which is approximated by (4.10). As will become apparent, the strategy for selecting the Gegenbauer reconstruction parameters $\alpha$ and $\beta$ is ideal for sparse data sets, that is, small $N$. For higher resolution, round-off error prohibits the Gegenbauer reconstruction method from converging for large $m$ and $\lambda$, so other parameter alternatives must be considered. We experimented with the functions

\begin{tabular}{||c|c|c|c|c|c||}
\hline \hline$\rho$ & $r_{0}$ & $\widetilde{r}_{0}^{(N)}$ & $\alpha$ & $\beta$ & $q_{T}=q_{R}^{1}$ \\
\hline \hline 0.25 & 0.781 & 0.809 & 0.0035 & 0.0625 & 0.998 \\
0.5 & 0.618 & 0.618 & 0.0313 & 0.177 & 0.984 \\
1 & 0.414 & 0.393 & 0.176 & 0.343 & 0.923 \\
2 & 0.236 & 0.249 & 0.412 & 0.410 & 0.837 \\
4 & 0.123 & 0.130 & 0.724 & 0.395 & 0.721 \\
\hline \hline
\end{tabular}

TABLE 5.1

Parameters $r_{0}, \widetilde{r}_{0}^{(N)}$ for $K=N=20, \alpha, \beta$ and $q_{T}=q_{R}^{1}$ for $\rho=0.25,0.5,1,2$ and 4 corresponding to the function (4.7).

\begin{tabular}{||c|c|c|c|c|c||}
\hline \hline$\rho$ & $r_{0}$ & $\widetilde{r}_{0}^{(N)}$ & $\alpha$ & $\beta$ & $q_{T}=q_{R}^{1}$ \\
\hline \hline 0.25 & 0.500 & 0.540 & 0.061 & 0.234 & 0.970 \\
0.5 & 0.382 & 0.405 & 0.163 & 0.335 & 0.929 \\
1 & 0.268 & 0.269 & 0.370 & 0.401 & 0.852 \\
2 & 0.172 & 0.185 & 0.566 & 0.412 & 0.780 \\
4 & 0.101 & 0.113 & 0.777 & 0.385 & 0.700 \\
\hline \hline
\end{tabular}

TABLE 5.2

Parameters $r_{0}, \widetilde{r}_{0}^{(N)}$ for $K=N=20, \alpha, \beta$ and $q_{T}=q_{R}^{1}$ for $\rho=0.25,0.5,1,2$ and 4 corresponding to the function (4.11).

defined by (4.7) and (4.11), which have singularities on imaginary and real axis. For 
these respective functions, Tables 5.1 and 5.2 compare the exact values of $r_{0}$ given by (3.3) and (4.12) to the values $\widetilde{r}_{0}^{(N)}$ (4.10). Also displayed are the optimized parameters $\alpha$ and $\beta$ corresponding to $\widetilde{r}_{0}^{(N)}$, which are computed by the least squares minimization method described in $\S 3$. Finally, the last column shows the minimum balanced values of $q_{T}=q_{R}^{1}$ which indicate the speed of theoretical exponential convergence of the resulting Gegenbauer reconstruction.

In the numerical experiments that follow, we consider the following four options for choosing the Gegenbauer parameters $\alpha$ and $\beta$ :

- Option 1: $\beta=0.25$ and $\alpha=1.31$ computed from the formula (3.1).

- Option 2: $\alpha=\beta=0.25$, as suggested in [14].

- Option 3: $\alpha=\beta=0.4$, also suggested in [14].

- Option 4: $\alpha$ and $\beta$ computed by the minimization of $\phi^{1}(\alpha, \beta)$ in (3.2).

These values (corresponding to option 4) are listed in Table 5.1 for the function (4.7) and in Table 5.2 for the function (4.11) for specific values of the parameter $\rho$.

Figure 5.1 compares the Gegenbauer and Fourier partial sum reconstruction of (4.7) for $\rho=1$ and (4.11) for $\rho=4$ based on the parameters chosen by option 4 . In each case we used $N=32$. The results for the Gegenbauer reconstruction, using the parameters suggested by our strategy are clearly Gibbs free and convergent.
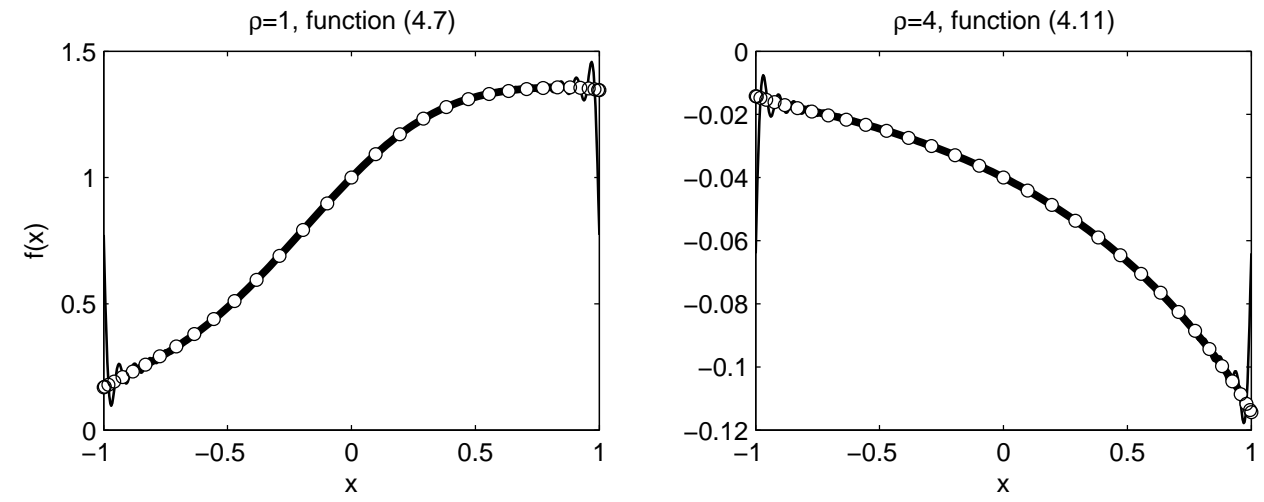

FIG. 5.1. A comparison of the Gegenbauer (denoted by circles) and Fourier partial sum reconstructions (thin lines) for (a) (4.7) with $\rho=1$ and (b) (4.11) with $\rho=4$. Original functions are plotted by thick lines. Here $N=32$, and $\alpha$ and $\beta$ chosen from option 4 .

The truncation (2.10) and regularization (2.12) errors can be viewed as competitive. Specifically, we observe that while the overall error converges for $\lambda, m \sim N$, the results of $q_{T}$ get worse as $m$ increases, and similarly $q_{R}^{1}$ (and $q_{R}^{2}$ ) will not decay as quickly for large $\lambda$. This behavior, illustrated in Figures 5.2 and 5.3, demonstrates why parameter optimization based on the analyticity of the underlying function is critical for the spectral convergence of the Gegenbauer reconstruction method, and why option 4 sometimes yields the only convergent approximation. The values of $\alpha$ and $\beta$ corresponding to option 4 and specific velues of the parameter $\rho$ are indicated on Figure 5.2 and 5.3 by white diamonds. This is further punctuated by the example given in (4.7) for $\rho \leq 0.5$. The Gegenbauer method is not convergent when $\alpha$ and $\beta$ are chosen according to option 1 , option 2 , or option 3 . Convergence is only observed when option 4 is used to pick the Gegenbauer parameters. Even in this case, as indicated by the ratio $q_{T}=q_{R}^{1}$ in Table 5.1 the convergence is quite slow and manifests itself only for large values of $N$. For example, both for $\rho=0.25$ and $\rho=0.5$, we 

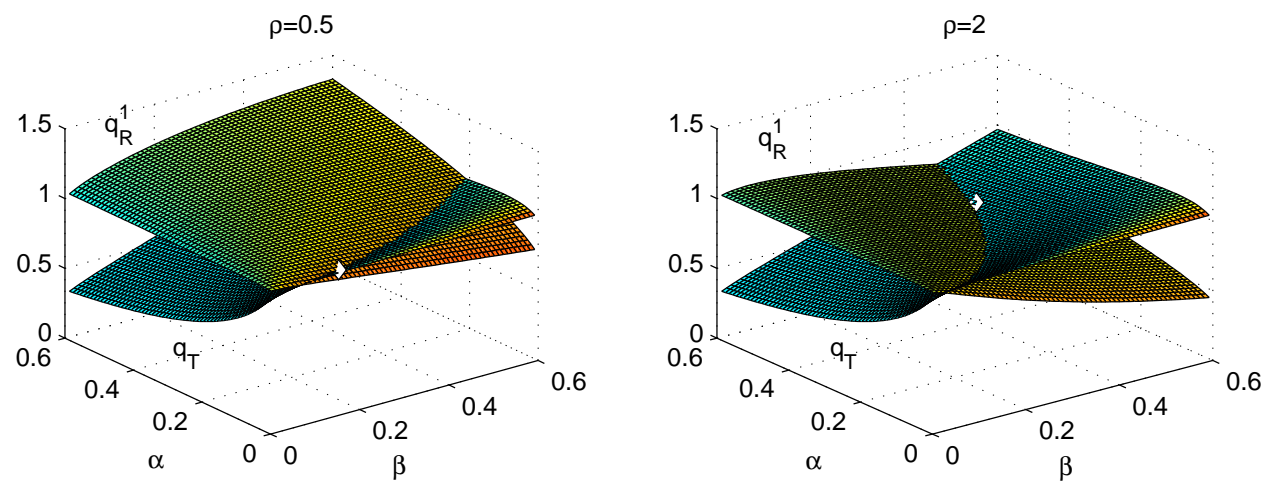

FIG. 5.2. A comparison of the "competing" regularization and truncation errors for the function (4.7) with (a) $\rho=0.5$ and (b) $\rho=2$.
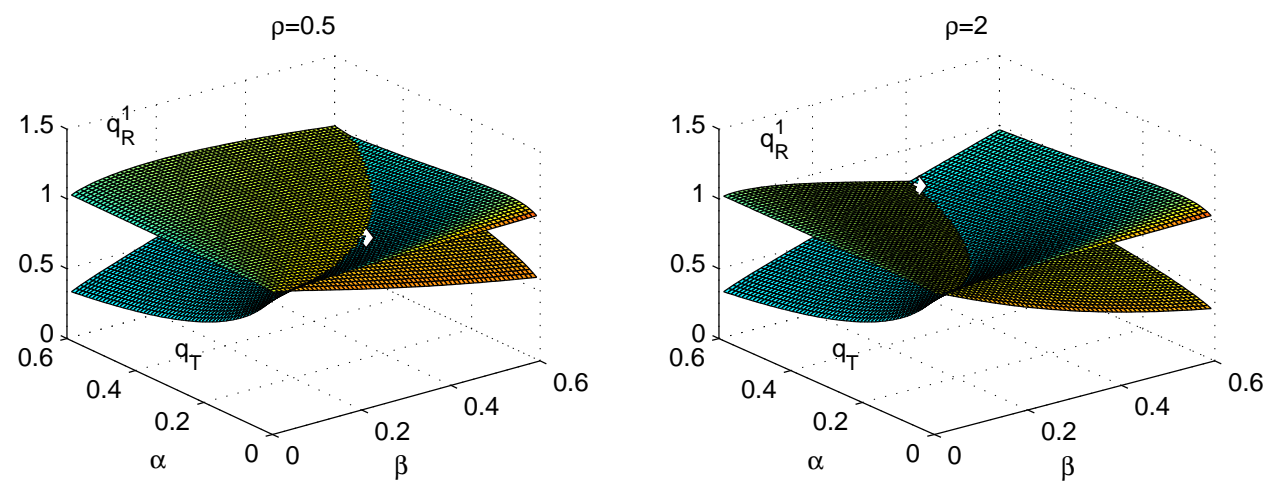

FIG. 5.3. A comparison of the "competing" regularization and truncation errors for the function (4.11) with (a) $\rho=0.5$ and (b) $\rho=2$.

require $N \geq 100$ for reasonable convergence and for $\rho=0.5 q_{T}=q_{R}^{1}$ only for values close to 1 . On the other hand, when $\rho=1,2$, or 4 , all of the parameter choosing options lead to exponential convergence. The fastest rate corresponds to option 4. Figure 5.4 (left) illustrates a comparison of the convergence rates of the Gegenbauer reconstruction method utilizing different strategies for determining $\alpha$ and $\beta$ for the example in (4.7) with $\rho=1$. A least squares fit of these errors is also provided. As displayed in Table 5.1, when $\rho=2$ the values of $\alpha$ and $\beta$ determined by option 2 and by option 4 are quite similar, leading to similar convergence rates for these two choices of $\alpha$ and $\beta$.

Similar conclusions are also valid for the example given in (4.11), although in this case we observe exponential convergence for all choices of $\alpha$ and $\beta$ and all values of $\rho$ listed in Table 5.2. The parameters chosen by option 4 yield the fastest rate of convergence with the most pronounced improvement occurring for large values of $\rho$. For illustration, the errors of the resulting Gegenbauer reconstruction corresponding to $\rho=4$ and $\alpha$ and $\beta$ chosen according to option 1, option 2, option 3, and option 4 are displayed in Figure 5.4 (right) along with the least squares fits of these errors.

We note that the results are shown for relatively sparse data sets, or small $N$. As $N$ and subsequently $\lambda=\alpha N$ and $m=\beta N$ increase, round-off error prohibits the 

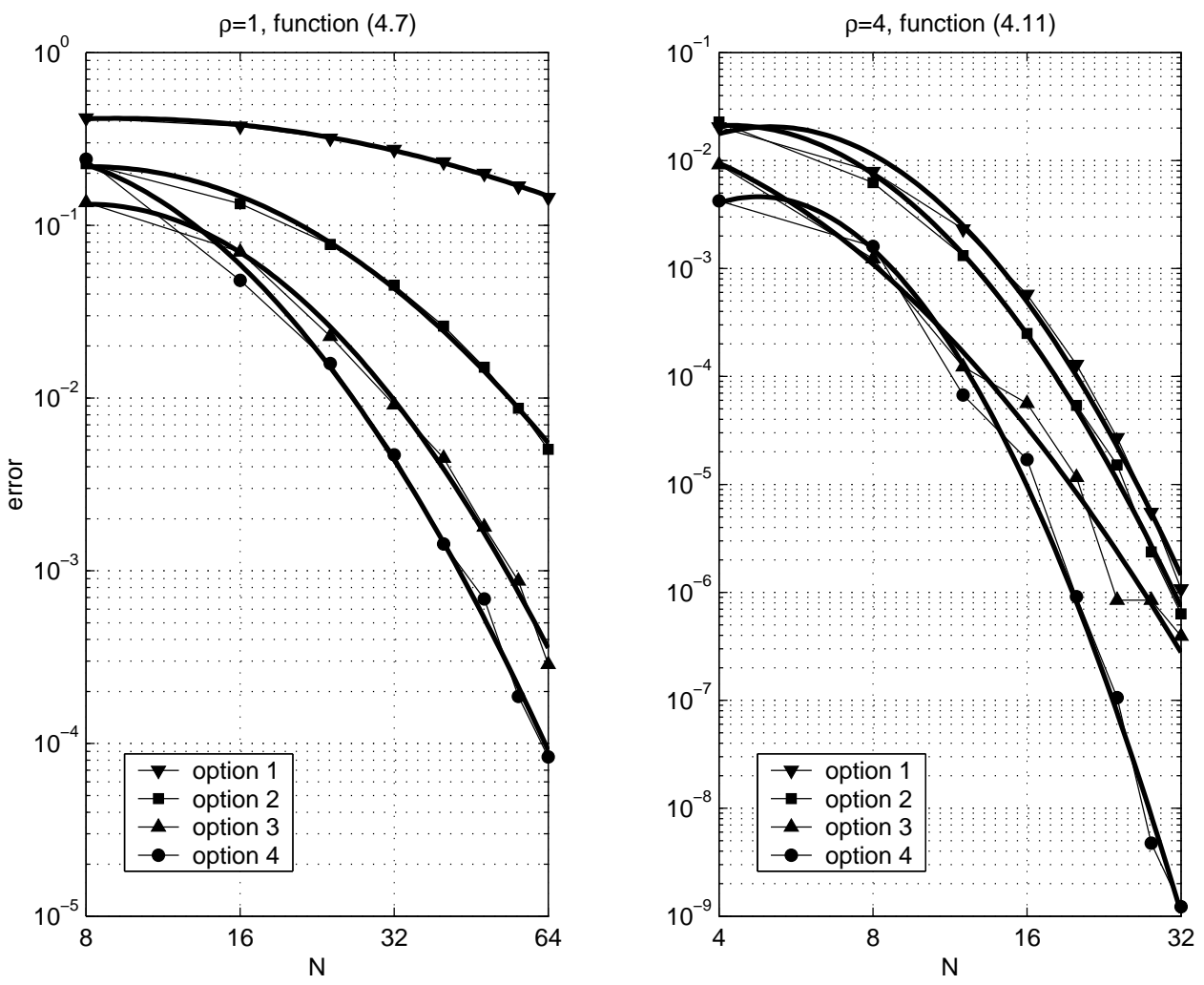

FIG. 5.4. Errors of the Gegenbauer reconstruction methods for $\rho=1$ and function (4.7) (left graph) and for $\rho=4$ and the function (4.11) (right graph) versus $N$ for different values of the parameters $\alpha$ and $\beta$. Least squares fits of these errors are also plotted by a thick solid lines.

convergence of the numerical Gegenbauer coefficients (2.4), and the overall convergence of the Gegenbauer reconstruction method will start to deteriorate. In fact it is is possible to observe the beginning of round-off error effects in Figure 5.4 (right), where option 3 for $N=28$ and $N=32$ yields errors that do not signify exponential convergence. The continuation of these numerical experiments for larger $N$ would yield round-off error for all options, and eventually the Gegenbauer reconstruction method would fail to converge at all. This problem can be alleviated by restricting the parameters $m$ and $\lambda$ by some upper bound where the effects of round-off errors can be controlled. These topics are discussed further in the recent paper [7].

6. Concluding remarks. The efficient resolution of a discontinuous function $f$ from its first $2 N+1$ Fourier coefficients by the Gegenbauer reconstruction method requires the determination of the parameters $\lambda=\alpha N$ and $m=\beta N$ of the Gegenbauer polynomials $C_{l}^{\lambda}$, and coefficients $\widehat{g}^{\lambda}(l), l=0,1, \ldots, m$. These parameters determine the speed of exponential convergence of the resulting reconstruction. However, they depend on the smoothness characteristics of the function $f$ and are usually not known in advance. In this paper we have developed a new technique to estimate these smoothness characteristics of the function $f$ from the Fourier partial sum $f_{N}(x)$. The smoothness of the function $f$ can be characterized by a parameter $r_{0}$ which corresponds to the 'size' of the ellipse for which the function $f$ has analytic continuation. 
Our technique of assessing $r_{0}$ is based on using the information provided by the Chebyshev coefficients $a_{k}^{(N)}$ corresponding to the partial Fourier sum $f_{N}(x)$ and utilizes the fundamental formula $\lim \sup _{k \rightarrow \infty}\left|a_{k}\right|^{1 / k}=r_{0}$, where $a_{k}$ are Chebyshev coefficients of $f$, as discussed in [9]. The approximation method for the smoothness parameter $r_{0}$ described in this paper is suitable for one dimensional problems, but due to its construction, we anticipate additional complexities in higher dimensions. Future work will address these issues. Once $r_{0}$ is computed, the optimal parameters $\alpha$ and $\beta$ are then determined by minimizing the objective function (3.2) which assumes that the convergence rates corresponding to the regularization and truncation errors of the Gegenbauer reconstruction are asymptotically equal. The effectiveness of this technique for sparse data sets is demonstrated by numerical experiments for discontinuous functions which have singularities on the real or imaginary axis. In the case of large data sets, the smoothness indicator can be combined with other error estimates to alleviate the effects of round-off errors.

The results given here and other recent investigations suggest that the Gegenbauer reconstruction method may be beneficial for a wide variety of problems, particularly in imaging applications, especially if it continues to produce evidence of satisfactory performance in noisy environments. This will be the topic of future investigations, as will smoothness parameter approximation in higher dimensions.

Acknowledgements. The authors wish to express their gratidude to the anonymous referees for their useful comments.

\section{REFERENCES}

[1] R. Archibald And A. Gelb, A method to reduce the Gibbs ringing artifact in MRI scans while keeping tissue boundary integrity, IEEE Trans. Medical Imaging, 21 (2002), pp. 305-319.

[2] R. Archibald and A. Gelb, Reducing the effects of noise in image reconstruction, J. Sci. Comput., 17 (2002), pp. 167-180.

[3] M. Abramowitz and I.S. Stegun, Handbook of Mathematical Functions with Formulas, Graphs, and Mathematical Tables, National Bureau of Standards, Washington, 1972.

[4] W. Cai, D. GotTlieb, And C.W. Shu, Essentially non-oscillatory spectral Fourier methods for shock wave calculations, Math. Comp., 52 (1989), pp. 389-410.

[5] P.J. DAvis, Methods of Numerical Integration, Academic Press, Ontario, San Diego, New York, 1984.

[6] K.S. EcKHOFF, Accurate reconstructions of functions of finite regularity from truncated series expansions, Math. Comp., 64 (1995), pp. 671-690.

[7] A. GeLB, On the reduction of round-off error for the Gegenbauer reconstruction method, J. Sci. Comp., 20(3) (2004), pp. 433-459.

[8] A. Gelb And E. TADMOR, Enhanced spectral viscosity approximations for conservation laws, Appl. Numer. Math., 33 (2000), pp. 3-21.

[9] D. Gottlieb and S.A. Orszag, Numerical Analysis of Spectral Methods: Theory and Applications, Society for Industrial and Applied Mathematics, Philadelphia, 1986.

[10] D. Gottlieb, B. Gustafsson, and P. Forssen, On the direct Fourier method for computer tomography, IEEE Trans. Medical Imaging, 19 (2000), pp. 223-233.

[11] D. Gottlieb and C.W. Shu, On the Gibbs phenomenon IV: Recovering exponential accuracy in a subinterval from a Gegenbauer partial sum of a piecewise analytic function, Math. Comp., 64 (1995), pp. 1081-1095.

[12] D. GotTlieb And C.W. Shu, On the Gibbs phenomenon and its resolution, SIAM Rev., 39 (1997), pp. 644-668.

[13] D. Gottuieb And C.W. Shu, A general theory for the resolution of the Gibbs phenomenon, Atti dei Convegni Lincei, 147 (1998), pp. 39-48.

[14] D. Gottlieb, C.W. Shu, A. Solomonoff, And H. Vandeven, On the Gibbs phenomenon I: recovering exponential accuracy from the Fourier partial sum of a nonperiodic analytic function, J. Comp. Appl. Math., 43 (1992), pp. 81-98. 
[15] Z. JACKIEwICZ, Determination of optimal parameters for the Chebyshev-Gegenbauer reconstruction method, SIAM J. Sci. Comput., 25 (2003/2004), pp. 1187-1198.

[16] Z. JACKIEWICZ AND B. ZUBIK-Kowal, Spectral collocation and waveform relaxation methods with Gegenbauer reconstruction for nonlinear conservation laws, submitted.

[17] B. Shizgal And J.H. Jung, Towards the resolution of the Gibbs phenomena, J. Comput. Appl. Math., 161 (2003), pp. 41-65.

[18] A. Zygmund, Trigonometrical Series, Dover Publications, New York, 1955. 